Z Research Square

\title{
Lysyl oxidase 473G/A (rs1800449) polymorphism influences the risk of cancer progression: a meta-analysis
}

\author{
Rungrawee Mongkolrob \\ Thammasat University \\ Phuntila Tharabenjasin \\ Thammasat University \\ Aporn Bualuang \\ Thammasat University \\ Noel Pabalan ( $\square$ noelpabalan@mail.com ) \\ Angeles University Foundation https://orcid.org/0000-0003-2069-5535
}

Research article

Keywords: Lysyl oxidase, LOX, 473G/A, rs1800449, polymorphism, cancer, meta-analysis

Posted Date: December 6th, 2019

DOI: https://doi.org/10.21203/rs.2.18129/v1

License: (c) (1) This work is licensed under a Creative Commons Attribution 4.0 International License. Read Full License 


\section{Abstract}

The genetics of cancer progression is important for devising optimal therapeutic strategies. Lysyl oxidase (LOX) is a cancer progression gene that has been studied enough to warrant synthesis of its primary data from association studies. However, reported associations between the LOX G473A (rs1800449) gene polymorphisms and cancer have been inconsistent prompting a meta-analysis so that we could obtain more precise estimates. Databases searches of the published literature yielded seven case-control studies. We calculated pooled odds ratios (ORs) and $95 \%$ confidence intervals (Cls) using four genetic models, homozygous (H), recessive (R), dominant (D) and codominant (C). Subgroup analysis was based on ethnicity and cancer type. Outlier analysis was used to examine sources of heterogeneity. Strength of evidence was based on high associative significance (expressed in terms of the Bayes Factor), consistency and magnitude of effects, homogeneity and robustness. $H / R$ outcomes exerted more associative effects than D/C results. Two reasons for these are: (i) $H / R$ analyses precluded outlier treatment; (ii) magnitude of $H / R$ (ORs of > 2.0) was twice that of $D / C$ (ORs > 1.0). All else being equal, significant pooled ORs in all genetic models had high significance $(\mathrm{Pa}<10-5)$. Strong evidence for associations were found in the outcomes for Asian and digestive cancers. In summary, the LOX rs1800449 polymorphism confers significant overall susceptibility, particularly in digestive cancers and places Asians at risk.

\section{Introduction}

Advanced cancer has been hypothesized to involve gene expression and/or inactivation of genes which are different from those in early tumorigenesis [1]. Understanding the role of these genes warrants a good grasp of primary research outcomes. In this study, we examined gene association effects and focus on cancer progression studies using the systematic synthesis approach. An important gene crucial to cancer progression is lysyl oxidase (LOX) which is a copper-dependent amine oxidase encoded by members of a five-gene family that includes LOX and four LOX-like proteins (LOXL1-4). Located on chromosome 5q23.1-q23, the LOX gene has seven exons that encode several functional domains of the LOX protein [2]. The LOX gene has an important single nucleotide polymorphism (SNP) in the open reading frame resulting in an Arg158GIn substitution in a highly conserved region (G473A, rs1800449). The GIn variant encoded by the minor A allele was shown to impair tumor suppressor ability compared with LOX wild type. Recent studies have shown that expression of LOX is increased in tumors or malignant cell lines of various cancers [3-7]. At the gene level, single-study reports of the LOX rs1800449 associations with cancer have not been consistent. It is thus opportune to statistically synthesize the findings of these studies using meta-analysis. Here, we examine the role of LOX rs1800449 SNP in the risk of cancer progression, which might guide potential future directions in cancer genetics. To obtain less ambiguous, clearer estimates of the role of SNPs in this investigation, we assessed the strength of evidence using various statistical and meta-analytical criteria. This study aims to highlight the genetic role of LOX rs1800449 in cancer progression and provide important information that could be useful in clinical decision making.

\section{Materials And Methods Selection of studies}

We searched MEDLINE using PubMed, Google Scholar and Science Direct for association studies as of August 17, 2019. The terms used were "Lysyl oxidase", "LOX", "polymorphism" and "cancer" as medical subject heading and text. References cited in the retrieved articles were also screened manually to identify additional eligible studies. Inclusion criteria were (1) case-control studies evaluating the association between LOX polymorphisms and cancer risk; (2) sufficient genotype frequency data presented to calculate the odds ratios (ORs) and $95 \%$ confidence intervals (Cls). Exclusion criteria were: (1) reviews; (2) studies whose control frequencies deviated from the Hardy-Weinberg Equilibrium (HWE); (3) not casecontrol; and (iv) unusable genotype data.

\section{Data extraction}

Two investigators (RM and NP) independently extracted data. A third investigator (PT) adjudicated disagreements until consensus was reached. The following information was obtained from each publication: first author's name, published year, country of origin, ethnicity, cancer type, study design, studies that matched their controls with cases and the criteria used, sample sizes and genotype frequencies.

\section{Methodological quality of the studies}

We used the Clark-Baudouin (CB) scale to evaluate methodological quality of the included studies [8]. The CB criteria include P-values, statistical power, and corrections for multiplicity, comparative sample sizes between cases and controls, genotyping methods and the HWE. In this scale, low, moderate and high have scores of $<5,5-6$ and $\geq 7$, respectively.

\section{Data distribution and power calculations}

Data distribution was assessed with the Shapiro-Wilks (SW) test using SPSS 20.0 (IBM Corp., Armonk, NY, USA). Gaussian (normal) distribution (P > 0.05 ) warranted descriptive expressions of mean \pm standard deviation (SD). Otherwise, the median (with interquartile range) was used. Using the G*Power program [9], we evaluated statistical power as its adequacy bolsters the level of associative evidence. Assuming an OR of 1.5 at a genotypic risk level of $a=0.05$ (two-sided), power was considered adequate at $\geq 80 \%$.

\section{HWE}


Using the application in https://ihg.gsf.de/cgi-bin/hw/hwa1.pl, we assessed the HWE and reported the P-value of the controls from the Pearson's goodness-of-fit $\chi^{2}$-square test. A P-value of $<0.05$ indicated deviation from the HWE. Deviations were found in six studies [10-15] thus were excluded from the analysis (Table S1). Table S2 accommodates a column that details the non-significance $(P>0.05)$ of the HWE-compliant studies.

\section{Data synthesis}

Cancer risks (ORs and 95\% Cls) were estimated for each study using the following genetic models: (i) homozygous [H] (ii) recessive [R] (iii) dominant [D], and (iv) codominant [C]. Comparing the effects on the same baseline, we calculated pooled ORs and $95 \%$ Cls. In addition to the overall analysis, we also examined two subgroups, Asians (3,834 cases/4,061 controls) and cancer type. The latter was stratified into digestive (1,453 cases/1,546 controls) and breast (935 cases/923 controls). Strength of evidence was assessed using three indicators: First, the magnitude of effects are higher or lower when the pooled ORs are farther from or closer to the OR value of 1.0 (null effect), respectively [16]. Second, the P-value is interpreted in terms of the Bayes Factor (BF), which is supported by evidence from both null and alternate hypotheses. In contrast, the P-value, by itself, addresses the null hypothesis only [17]. Thus, P-values of 0.05 and 0.001 correspond to the minimum BFs of $\geq 0.15$ and 0.005 , indicating moderate and strong (to very strong) evidence, respectively [18]. The BF rests on the likelihood paradigm [19], where strength of the hypotheses rests on the data [17]. Thus, the likelihoods between the absence (null hypothesis) and presence (alternate hypothesis) of association of LOX with cancer are compared. Third, homogeneity is preferred to heterogeneity, but heterogeneity is unavoidable [20]. The reason for this preference is that conclusions made in the milieu of homogeneity have greater evidential strength than those that are heterogeneously derived. Thus, presence of heterogeneity between studies was estimated with the $\chi^{2}$-based $Q$ test [21], with threshold of significance set at $P^{b}<0.10$. Heterogeneity was quantified with the $I^{2}$ statistic which measures variability between studies [22]. $I^{2}$ values of $>50 \%$ indicate more variability than those $\leq 50 \%$ with $0 \%$ indicating zero heterogeneity (homogeneity). Evidence of functional similarities in population features of the studies warranted using the fixed-effects (Fe) model [23], otherwise the random-effects $(\mathrm{Re})$ model [24] was used. Sensitivity analysis, which involves omitting one study at a time and recalculating the pooled OR, was used to test for robustness of the summary effects. We did not assess publication bias because none of the comparisons had $\geq 10$ studies. Less than this number presents low sensitivity of the publication bias tests [25]. Except for heterogeneity estimation [21], two-sided P-values of $\leq 0.05$ were considered significant. All associative outcomes were Bonferroni-corrected. Data for the meta-analysis were analyzed using Review Manager 5.3 (Cochrane Collaboration, Oxford, England), SIGMASTAT 2.03, and SIGMAPLOT 11.0 (Systat Software, San Jose, CA).

\section{Results}

\section{Characteristics of the included studies}

Figure 1 outlines the selection process in a PRISMA (Preferred Reporting Items for Systematic Reviews and Meta-Analyses)-sanctioned flowchart [26]. A total of 107 citations were identified from the initial search, the screening of which yielded 20 full-text articles. From the 20,13 were excluded for not conforming to our inclusion criteria. Manual search of the references from the publications yielded no additional articles. Table 1 lists the seven articles included in this meta-analysis [27-33]. One article [32] covered two cancer types (lung and colorectal) which was treated as two studies. Subjects were all Asians except in two publications [27, 29]. Three articles focused on the breast [27, 29, 30]. Four articles [28, 31, 33, 32] examined digestive cancers (oral, gastric and colorectal). The normally distributed (SW: $\mathrm{P}=0.139$ ) CB scores showed a mean \pm SD of $6.00 \quad \pm 1.16$, indicating that the methodological quality of the component studies was moderate. Table S2 shows the quantitative traits of the eight included studies. Sample sizes ranged from 299 to 1,273. One study [28] was statistically adequate (94.4\%). The PRISMA checklist provides detailed description of this meta-analysis (Table S3).

Table 1

Characteristics of the included articles that examined Lysyl oxidase (rs1800449) associations with cancer

\begin{tabular}{|lllllllllc|}
\hline & First author & {$[R]$} & Year & Country & Ethnic Group & Cancer type & Study design & Matching based on \\
\hline & & & & & & & & \\
\hline 1 & Friesenhengst & {$[27]$} & 2014 & Austria & Caucasian & Breast & HB & residence \\
\hline 2 & Gao & {$[28]$} & 2014 & China & Asian & Colorectal & HB & age, sex \\
\hline 3 & Min & {$[29]$} & 2009 & USA & AA & Breast & PB & age, geography \\
\hline 4 & Ren & {$[30]$} & 2011 & China & Asian & Breast & HB & age \\
\hline 5 & Shieh & {$[31]$} & 2009 & Taiwan & Asian & Oral & NM & NM \\
\hline 6 & Wang & {$[32]$} & 2016 & China & Asian & Lung, colorectal & PB & age, sex, residence \\
\hline 7 & Yoon & {$[33]$} & 2011 & South Korea & Asian & Gastric & PB & NM \\
\hline
\end{tabular}

\section{Overall and subgroup analysis}


Tables 2 and 3 present 15 significant $\left(P^{a}<0.05\right)$ outcomes, $14(93.3 \%)$ of which had $P^{a}$-values that reached $<10^{-5}$ indicating both consistency and strong effects. Table 2 shows all the Fe results with three important features: (i) initial Fe precluded outlier treatment; (ii) H/R outcomes (ORs 2.3-2.5) had twice the magnitude of effect over the D/C results (ORs 1.4-1.6) and (iii) a strong case of LOX rs 1800449 associations with cancer based on the core outcomes. Judged on the basis of high significance $\left(\mathrm{P}^{\mathrm{a}}<10^{-5}\right)$ and homogeneity $\left(\mathrm{I}^{2}=0 \%\right)$, we identified four core outcomes $(\lceil)$, which were ranked based on the number of studies $(n)$, with elevated $n$ indicating a higher rank. Thus, the Asian outcome $(n=6)$ in the $C$ model was ranked first (OR 1.53, 95\% Cl 1.38-1.70, $\mathrm{P}^{\mathrm{a}}<10^{-5}$ ). This core finding (Table 2) is the same as the overall outcome seen in the PSO panel for the $\mathrm{C}$ model (Table 3), with all Asian subjects. Table 2 shows the subordinate in rank results with a collective outcome that identified significant associations in the digestive cancers (R/D/C: ORs 1.45-2.34, 95\% Cls 1.24-3.15, $\mathrm{P}^{\mathrm{a}}<10^{-5}$ ). The $\mathrm{P}^{\mathrm{a}}$-values in these outcomes showed that in $\mathrm{BF}$ terms, this indicates strong evidence of association, underpinned by study compatibility. Of note, sensitivity treatment rendered robustness to all the highly significant outcomes (Table 4).

Table 2

Summary associations of all fixed-effects outcomes between Lysyl oxidase (rs1800449) and cancer

\begin{tabular}{|c|c|c|c|c|c|c|}
\hline \multirow[b]{2}{*}{ Comparison } & \multicolumn{3}{|c|}{ Test of association } & \multicolumn{3}{|c|}{ Test of heterogeneity } \\
\hline & $\mathrm{n}$ & OR & $95 \% \mathrm{Cl}$ & $\mathrm{P}^{\mathrm{a}}$ & $\mathrm{P}^{\mathrm{b}}$ & $\mathrm{I}^{2}(\%)$ \\
\hline \multicolumn{7}{|l|}{ All } \\
\hline $\mathrm{H}$ & 8 & 2.28 & $1.79-2.90$ & $10^{-5}$ & 0.19 & 30 \\
\hline $\mathrm{R}$ & 8 & 2.26 & $1.79-2.86$ & $10^{-5}$ & 0.13 & 37 \\
\hline \multicolumn{7}{|l|}{ Asian } \\
\hline $\mathrm{H}$ & 6 & 2.47 & $1.90-3.20$ & $10^{-5}$ & 0.27 & 22 \\
\hline $\mathrm{R}$ & 6 & 2.43 & $1.89-3.14$ & $10^{-5}$ & 0.17 & 36 \\
\hline $\mathrm{C}\lceil$ & 6 & 1.53 & $1.38-1.70$ & $10^{-5}$ & 0.43 & 0 \\
\hline \multicolumn{7}{|c|}{ Digestive cancers* } \\
\hline $\mathrm{H}$ & 4 & 2.32 & $1.71-3.15$ & $10^{-5}$ & 0.27 & 23 \\
\hline $\mathrm{R}\lceil$ & 4 & 2.34 & $1.74-3.15$ & $10^{-5}$ & 0.58 & 0 \\
\hline $\mathrm{D}\lceil$ & 4 & 1.45 & $1.24-1.68$ & $10^{-5}$ & 0.52 & 0 \\
\hline $\mathrm{C}\lceil$ & 4 & 1.48 & $1.31-1.68$ & $10^{-5}$ & 0.61 & 0 \\
\hline \multicolumn{7}{|c|}{ Breast cancer } \\
\hline $\mathrm{H}$ & 3 & 1.57 & $0.95-2.58$ & 0.08 & 0.52 & 0 \\
\hline $\mathrm{R}$ & 3 & 1.37 & $0.84-2.24$ & 0.21 & 0.59 & 0 \\
\hline \multicolumn{7}{|c|}{ H: homozygous; R: recessive; D: dominant; C: codominant; l core findings (judged on } \\
\hline \multicolumn{7}{|c|}{ the basis of high associative significance $\left(\mathrm{P}^{\mathrm{a}}<10^{-5}\right.$ and homogeneity: $\left.\mathrm{I}^{2}=0 \%\right)$; : number } \\
\hline \multicolumn{7}{|c|}{ of studies; OR: odds ratio; Cl: confidence interval; $\mathrm{P}^{\mathrm{a}}$ : P-value for association; $\mathrm{P}^{\mathrm{b}}$ : P-value } \\
\hline \multicolumn{7}{|c|}{ for heterogeneity; $\mathrm{l}^{2}$ is a measure of variability; All comparisons are fixed-effects with their } \\
\hline
\end{tabular}


Table 3

Outlier outcomes of the Lysyl oxidase (rs1800449) and cancer

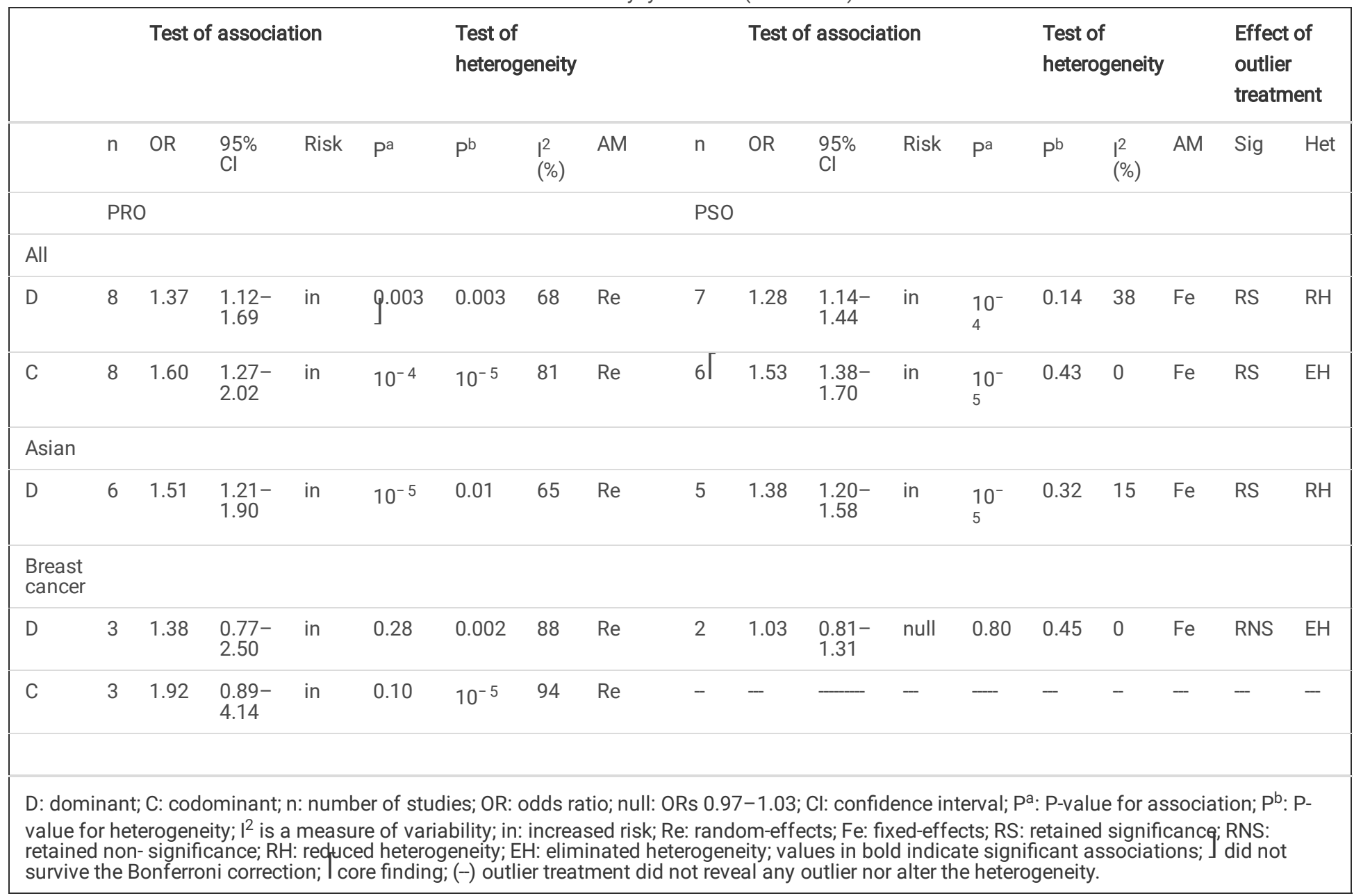


Table 4

Sensitivity analysis of significant outcomes

\begin{tabular}{|c|c|c|c|}
\hline Comparison Genetic model & os & $P^{a}$-value & Sensitivity outcome \\
\hline \multicolumn{4}{|l|}{ All } \\
\hline $\mathrm{H}$ & - & $<10^{-5}$ & Robust \\
\hline $\mathrm{R}$ & - & $<10^{-5}$ & Robust \\
\hline D & PRO & 0.003 & Robust \\
\hline C & PRO & $<10^{-4}$ & Robust \\
\hline D & PSO & $<10^{-4}$ & Robust \\
\hline C & PSO & $<10^{-5}$ & Robust \\
\hline \multicolumn{4}{|l|}{ Asian } \\
\hline $\mathrm{H}$ & - & $<10^{-5}$ & Robust \\
\hline $\mathrm{R}$ & - & $<10^{-5}$ & Robust \\
\hline D & PRO & $<10^{-5}$ & Robust \\
\hline D & PSO & $<10^{-5}$ & Robust \\
\hline cl & - & $<10^{-5}$ & Robust \\
\hline \multicolumn{4}{|l|}{ Digestive cancers* } \\
\hline $\mathrm{H}$ & - & $<10^{-5}$ & Robust \\
\hline $\mathrm{R}\lceil$ & - & $<10^{-5}$ & Robust \\
\hline $\mathrm{D} \Gamma$ & - & $<10^{-5}$ & Robust \\
\hline$c\lceil$ & - & $<10^{-5}$ & Robust \\
\hline \multicolumn{4}{|c|}{ H: homozygous; R: recessive; D: dominant; C: codominant; [ core findings; } \\
\hline
\end{tabular}

\section{Impact and mechanism of outlier treatment}

The impact of outlier treatment is best seen in Table 3, which is bisected into left and right panels, indicating PRO and PSO, respectively. A salient feature of this table is that all the PRO outcomes were Re-derived (analysis model column), concomitant with $\mathrm{I}^{2}$ values of $65 \%-94 \%$. On the other hand, all PSO results were Fe-derived $\left(\mathrm{I}^{2}=0 \%-38 \%\right)$. The mechanism of outlier treatment for the $\mathrm{C}$ model in the overall analysis is visualized in Figs. 2-4. Figure 2 shows the PRO forest plot, where the pooled outcome indicated significant increased risk $\left(\mathrm{OR} 1.60,95 \% \mathrm{Cl} 1.27-2.02, \mathrm{P}^{\mathrm{a}}<10^{-4}\right)$ and heterogeneity $\left(\mathrm{P}^{\mathrm{b}}<10^{-5}, \mathrm{I}^{2}=81 \%\right)$. The Galbraith plot identified two studies $[27,29]$ as the outliers, located above and below the +2 and -2 confidence limits (Fig. 3). In Fig. 4, the PSO outcome (outliers omitted) shows eliminated heterogeneity $\left(P^{b}=0.43, I^{2}=0 \%\right)$; modulated increased risk (OR 1.53, 95\% Cl 1.38-1.70) and retained significance $\left(\mathrm{P}^{\mathrm{a}}<10^{-5}\right)$. This operation is numerically summarized in Table 3.

\section{Discussion}

\section{Summary of findings}

The main findings of this study presented layers of evidence pointing to strong associations between LOX rs1800449 and risk of cancer progression. These layers included consistency of effects (the significant ORs were $>1.0$ across all comparisons) and significance (93.3\% of the comparisons had $\mathrm{P}^{\mathrm{a}}<10^{-5}$ ). Such level of significance enabled the outcomes to survive the Bonferroni correction attesting to their stability (an added layer) and minimizing the risk of type 1 error (false-positives). The outcomes that have met the criteria for strong evidence $\left(P^{\mathrm{a}}<10^{-5}\right.$ and $\left.\mathrm{I}^{2}=0 \%\right)$ were found in the $\mathrm{C}$ model (Asian) and the R/D/C models (digestive cancers) which comprised our core findings. 
The initial homogeneity of these Fe-derived core outcomes that precluded outlier treatment compel without the necessity of outlier treatment. One purpose of biomedical meta-analysis is subject the overall summary effects to meta-analytical procedures (outlier and sensitivity treatments, subgrouping) in order to test for their stability, consistency and robustness (another added layer). These features have for the most part, characterized our findings. Furthermore, ethnicity and subgrouping by cancer type did not materially alter the overall results, both in terms of direction of association and statistical significance. The lack of material differences (all the significant outcomes indicated increased risks) across comparisons suggests consistency of our results. Our digestive cancer findings, in particular, were noteworthy for their high significance and homogeneity in most genetic models. These significant findings underpin the importance of subgrouping in evaluating risk of disease polymorphisms. Primary study findings about the roles of genetic variants in cancer were not only conflicting, they have also been proven difficult to replicate. These maybe due to limited statistical power, heterogeneous subjects, presence of confounders and population stratification [8].

\section{Comparison with a previous meta-analysis}

The previous meta-analysis [28] and ours each comprised of seven papers; but only two [30,33] were common in both. These differences in the five included studies between the two meta-analyses impacted upon the study design with differential subgroupings (ethnicity and cancer type in ours versus Asian ethnicity in Gao et al [28]). The eventual findings also differed despite use of standard genetic models in both meta-analyses. The present meta-analysis involved the following protocols that were not performed previously: First, we formally evaluated the methodological quality of the included studies using CB. This facilitated better understanding of the quality of the source materials. Second, we used the $\mathrm{I}^{2}$ metric in assessing variability of the studies, providing a clearer picture of the magnitude of heterogeneity of the summary outcomes or lack of it. Third, we confined our analysis to HWE-compliant studies which minimized issues of genotyping error. Lastly, we performed subgrouping by cancer type, which unmasked specific tumor sites associated with LOX rs1800449. In the overall analysis, associative outcomes between the previous and ours by genetic model are as follows: (i) H: 1.5-fold versus 2.3-fold; (ii) R: 2.1-fold versus 2.3-fold; (iii) D: both at 1.4-fold; (iv) C: 1.3-fold versus 1.6-fold. These numbers indicate modulated effects in the previous meta-analysis compared to ours in the H/R/C models but not in the D model. Heterogeneity of these values did not differ between the two meta-analyses. The two insights that we offer from our meta-analysis are: (i) attention to study quality and (ii) differential findings from subgroup analysis by cancer type.

\section{The LOX gene and LOX protein in cancer}

Metastasis is the last stage of cancer progression that warrants a good understanding of its genetic etiology. The role of LOX in cancer metastasis has rendered this gene an appealing therapeutic target [34]. The multiple roles of LOX in cancer include, cell motility/migration/differentiation, cell adhesion, chemotaxis and gene transcription regulation [35]. These diverse roles open the possibilities for a significant role of LOX in cancer therapy [36]. Therapeutic targeting of LOX in the preclinical milieu involving inhibitors and function-blocking antibodies to prevent metastasis have been reportedly effective [37]. However, the contrasting roles of the LOX gene as tumor suppressor and metastatic progressor have been reported in the literature [38].

\section{Strengths and limitations}

Interpreting our meta-analysis findings are better contextualized in terms of their strengths and limitations. The limitation is that majority (84\%) of the studies had Asian subjects indicating under-representation of other ethnic groups. We had one study each for non-Hispanic Caucasians [27] and African-Americans [29]. Future studies warrant inclusion of more subjects with these ethnicities. Despite this limitation, the following strengths bolster confidence in our findings as they not only add to the epidemiological, clinical and statistical combinability of the studies, but also minimize bias and underpin a strong magnitude of association. (i) Our criteria of including only HWE-compliant studies effectively controlled for genotyping error and thus minimized methodological weaknesses in our study [39]. (ii) Most controls were uniformly defined (healthy or cancer-free); (iii) most tissue sources were blood; (iv) in most of the articles (five [27-30, 32] of seven or 71\%), controls were matched with cases, with 80\% (four [28-30, 32] of five) based on age. (v) Six [27-29,32,33] of the eight studies (75\%) had sample sizes of $\geq 500$; (vi) of the 20 comparisons, 15 (75\%) were statistically significant, $14(93.3 \%)$ of which with extreme P-values $\left(<10^{-5}\right)$. (vii) Outlier treatment proved effective in erasing heterogeneity in the $\mathrm{C}$ model of the core finding and (viii) sensitivity analysis rendered robustness to all significant outcomes.

\section{Conclusion}

In conclusion, this study presents meta-analytic evidence of the significant association between LOX rs1800449 and increased cancer risk. The observed associations here suggest that this polymorphism might be a useful susceptibility cancer marker. This marker potential may apply more for digestive system cancers than for breast cancer; more for Asians than other ethnic groups. However, a single locus effect on cancer will likely be small given the involvement of other factors such as gene-gene and gene-environment interactions. All seven publications focused only on LOX. Four $[28,30,32,33]$ of the seven articles $(57.1 \%)$ mentioned gene-environment interaction but did not provide data that could have been useful in further analysis. More studies that explore other cancer types and based on sample sizes commensurate with detection of small genotypic risks should allow more definitive conclusions about the association of LOX rs1800449 polymorphism and cancer.

\section{Abbreviations}



AA
African-American
AM
analysis model
BF
Bayes Factor
C
codominant
CB
Clark-Baudouin
$\mathrm{Cl}$
confidence interval
D
dominant
$\mathrm{EH} \quad$ eliminated heterogeneity
Fe fixed-effects
$\mathrm{H} \quad$ homozygous
HB hospital-based
Het heterogeneity
HWE Hardy-Weinberg Equilibrium
$R \quad$ measure of variability
in increased risk
LOX Lysyloxidasegene
LOX Lysyl oxidase protein
maf minor allele frequency
$\mathrm{n} \quad$ number of studies
NM not mentioned
OR odds ratio
OS outlier status
$P^{a} \quad P$-value for association
$P^{b} \quad P$-value for heterogeneity
PB population-based
PRISMA Preferred Reporting Items for Systematic Reviews and Meta-Analyses
PRO pre-outlier
PSO post-outlier
R recessive
Re random-effects
$\mathrm{RH} \quad$ reduced heterogeneity
RNS retained non-significance
RS retained significance
Sig significance 
SW Shapiro-Wilks

USA

United States of America

\section{Declarations}

\section{-Ethics approval and consent to participate}

not applicable

\section{-Consent for publication}

not applicable

\section{-Availability of data and material}

in supporting information

\section{-Competing interests}

The authors declare that they have no competing interests

\section{-Funding}

this study was unfunded

\section{-Authors' contributions}

Conceptualization - RM, NP, PT

Data extraction and analysis - RM, NP, PT

Validation - RM, PT, NP

Methodology - PT, NP

Software - NP

Writing - original draft - RM, NP, PT

Writing - review \& editing - RM, NP, PT

\section{-Acknowledgements}

not applicable

\section{References}

1. Rinker-Schaeffer CW, O'Keefe JP, Welch DR, Theodorescu D. Metastasis suppressor proteins: discovery, molecular mechanisms, and clinical application. Clinical cancer research : an official journal of the American Association for Cancer Research. 2006;12(13):3882-9. doi:10.1158/1078-0432.CCR-06-1014.

2. Csiszar K, Mariani TJ, Gosin JS, Deak SB, Boyd CD. A restriction fragment length polymorphism results in a nonconservative amino acid substitution encoded within the first exon of the human lysyl oxidase gene. Genomics. 1993;16(2):401-6. doi:10.1006/geno.1993.1203.

3. Kannan K, Amariglio N, Rechavi G, Jakob-Hirsch J, Kela I, Kaminski N et al. DNA microarrays identification of primary and secondary target genes regulated by p53. Oncogene. 2001;20(18):2225-34. doi:10.1038/sj.onc.1204319.

4. Montel V, Huang TY, Mose E, Pestonjamasp K, Tarin D. Expression profiling of primary tumors and matched lymphatic and lung metastases in a xenogeneic breast cancer model. The American journal of pathology. 2005;166(5):1565-79. doi:10.1016/S0002-9440(10)62372-3. 
5. Rost T, Pyritz V, Rathcke IO, Gorogh T, Dunne AA, Werner JA. Reduction of LOX- and LOXL2-mRNA expression in head and neck squamous cell carcinomas. Anticancer research. 2003;23(2B):1565-73.

6. Tsuchiya MI, Okuda H, Takaki Y, Baba M, Hirai S, Ohno S et al. Renal cell carcinoma- and pheochromocytoma-specific altered gene expression profiles in VHL mutant clones. Oncology reports. 2005;13(6):1033-41.

7. Uzel MI, Shih SD, Gross H, Kessler E, Gerstenfeld LC, Trackman PC. Molecular events that contribute to lysyl oxidase enzyme activity and insoluble collagen accumulation in osteosarcoma cell clones. Journal of bone and mineral research : the official journal of the American Society for Bone and Mineral Research. 2000;15(6):1189-97. doi:10.1359/jbmr.2000.15.6.1189.

8. Clark MF, Baudouin SV. A systematic review of the quality of genetic association studies in human sepsis. Intensive Care Med. 2006;32(11):170612.

9. Faul F, Erdfelder E, Lang AG, Buchner A. G*Power 3: a flexible statistical power analysis program for the social, behavioral, and biomedical sciences. Behavior research methods. 2007;39(2):175-91.

10. Bu M, Li L, Zhang Y, Xu Y, An S, Hou F et al. Lysyl oxidase genetic variants affect gene expression in cervical cancer. DNA and cell biology. 2014;33(11):787-92. doi:10.1089/dna.2014.2490.

11. Han S, Feng S, Yuan G, Dong T, Gao D, Liang G et al. Lysyl oxidase genetic variants and the prognosis of glioma. APMIS : acta pathologica, microbiologica, et immunologica Scandinavica. 2014;122(3):200-5. doi:10.1111/apm.12133.

12. Liu Y, Lv B, He Z, Zhou Y, Han C, Shi G et al. Lysyl oxidase polymorphisms and susceptibility to osteosarcoma. PloS one. 2012;7(7):e41610. doi:10.1371/journal.pone.0041610.

13. Shi W, Yang B, Li X, Sun S, Wang L, Jiao S. The effect of lysyl oxidase polymorphism on susceptibility and prognosis of nonsmall cell lung cancer. Tumour biology : the journal of the International Society for Oncodevelopmental Biology and Medicine. 2012;33(6):2379-83. doi:10.1007/s13277-012-0501-5.

14. Wang X, Cong JL, Qu LY, Jiang L, Wang Y. Association between lysyl oxidase G473A polymorphism and ovarian cancer in the Han Chinese population. The Journal of international medical research. 2012;40(3):917-23.

15. Wu J, Cai C, Tong D, Hou H. Lysyl oxidase G473A polymorphism is associated with increased risk of ovarian cancer. Genetic testing and molecular biomarkers. 2012;16(8):915-9. doi:10.1089/gtmb.2011.0374.

16. Chen $\mathrm{H}$, Cohen P, Chen S. How Big is a Big Odds Ratio? Interpreting the Magnitudes of Odds Ratios in Epidemiological Studies. Communications in Statistics-Simulation and Computation. 2010;39:860-4.

17. Goodman SN. Introduction to Bayesian methods I: measuring the strength of evidence. Clinical trials. 2005;2(4):282-90; discussion 301-4, 64-78. doi:10.1191/1740774505cn098oa.

18. Goodman SN. Toward evidence-based medical statistics. 2: The Bayes factor. Annals of internal medicine. 1999;130(12):1005-13. doi:10.7326/0003-4819-130-12-199906150-00019.

19. Royall R. Statistical evidence: The likelihood paradigm. London: Chapman and Hall; 1997.

20. Higgins JP. Commentary: Heterogeneity in meta-analysis should be expected and appropriately quantified. International journal of epidemiology. 2008;37(5):1158-60. doi:10.1093/ije/dyn204.

21. Higgins JP, Thompson SG, Deeks JJ, Altman DG. Measuring inconsistency in meta-analyses. Bmj. 2003;327(7414):557-60.

22. Higgins JP, Thompson SG. Quantifying heterogeneity in a meta-analysis. Stat Med. 2002;21(11):1539-58.

23. Mantel N, Haenszel W. Statistical aspects of the analysis of data from retrospective studies of disease. J Natl Cancer Inst. 1959;22(4):719-48.

24. DerSimonian R, Laird N. Meta-analysis in clinical trials. Control Clin Trials. 1986;7(3):177-88.

25. loannidis JP, Trikalinos TA. The appropriateness of asymmetry tests for publication bias in meta-analyses: a large survey. CMAJ : Canadian Medical Association journal = journal de l'Association medicale canadienne. 2007;176(8):1091-6. doi:10.1503/cmaj.060410.

26. Moher D, Liberati A, Tetzlaff J, Altman DG, Group P. Preferred reporting items for systematic reviews and meta-analyses: the PRISMA statement. Journal of clinical epidemiology. 2009;62(10):1006-12. doi:10.1016/j.jclinepi.2009.06.005.

27. Friesenhengst A, Pribitzer-Winner T, Schreiber M. Association of the G473A polymorphism and expression of lysyl oxidase with breast cancer risk and survival in European women: a hospital-based case-control study. PloS one. 2014;9(8):e105579. doi:10.1371/journal.pone.0105579.

28. Gao X, Zhang S, Zhu Z. Lysyl oxidase rs1800449 polymorphism and cancer risk among Asians: evidence from a meta-analysis and a casecontrol study of colorectal cancer. Molecular genetics and genomics : MGG. 2015;290(1):23-8. doi:10.1007/s00438-014-0896-3.

29. Min C, Yu Z, Kirsch KH, Zhao Y, Vora SR, Trackman PC et al. A loss-of-function polymorphism in the propeptide domain of the LOX gene and breast cancer. Cancer research. 2009;69(16):6685-93. doi:10.1158/0008-5472.CAN-08-4818.

30. Ren J, Wu X, He W, Shao J, Cheng B, Huang T. Lysyl oxidase 473 G>A polymorphism and breast cancer susceptibility in Chinese Han population. DNA and cell biology. 2011;30(2):111-6. doi:10.1089/dna.2010.1098.

31. Shieh TM, Tu HF, Ku TH, Chang SS, Chang KW, Liu CJ. Association between lysyl oxidase polymorphisms and oral submucous fibrosis in older male areca chewers. Journal of oral pathology \& medicine : official publication of the International Association of Oral Pathologists and the American Academy of Oral Pathology. 2009;38(1):109-13. doi:10.1111/j.1600-0714.2008.00695.x.

Page 10/13 
32. Wang G, Shen Y, Cheng G, Bo H, Lin J, Zheng M et al. Lysyl Oxidase Gene G473A Polymorphism and Cigarette Smoking in Association with a High Risk of Lung and Colorectal Cancers in a North Chinese Population. International journal of environmental research and public health. 2016;13(7). doi:10.3390/ijerph13070635.

33. Yoon JH, Park JK, Kang YH, Park YK, Nam SW, Lee JY et al. Lysyl oxidase G473A polymorphism is closely associated with susceptibility to gastric cancer in a South Korean population. APMIS : acta pathologica, microbiologica, et immunologica Scandinavica. 2011;119(11):762-8. doi:10.1111/j.1600-0463.2011.02802.x.

34. Perryman L, Erler JT. Lysyl oxidase in cancer research. Future oncology. 2014;10(9):1709-17. doi:10.2217/fon.14.39.

35. Nishioka T, Eustace A, West C. Lysyl oxidase: from basic science to future cancer treatment. Cell structure and function. 2012;37(1):75-80.

36. Siddikuzzaman, Grace VM, Guruvayoorappan C. Lysyl oxidase: a potential target for cancer therapy. Inflammopharmacology. 2011;19(3):117-29. doi:10.1007/s10787-010-0073-1.

37. Barker HE, Cox TR, Erler JT. The rationale for targeting the LOX family in cancer. Nature reviews Cancer. 2012;12(8):540-52. doi:10.1038/nrc3319.

38. Payne SL, Hendrix MJ, Kirschmann DA. Paradoxical roles for lysyl oxidases in cancer-a prospect. Journal of cellular biochemistry. 2007;101(6):1338-54. doi:10.1002/jcb.21371.

39. Thakkinstian A, McElduff P, D'Este C, Duffy D, Attia J. A method for meta-analysis of molecular association studies. Stat Med. 2005;24(9):1291306.

Figures

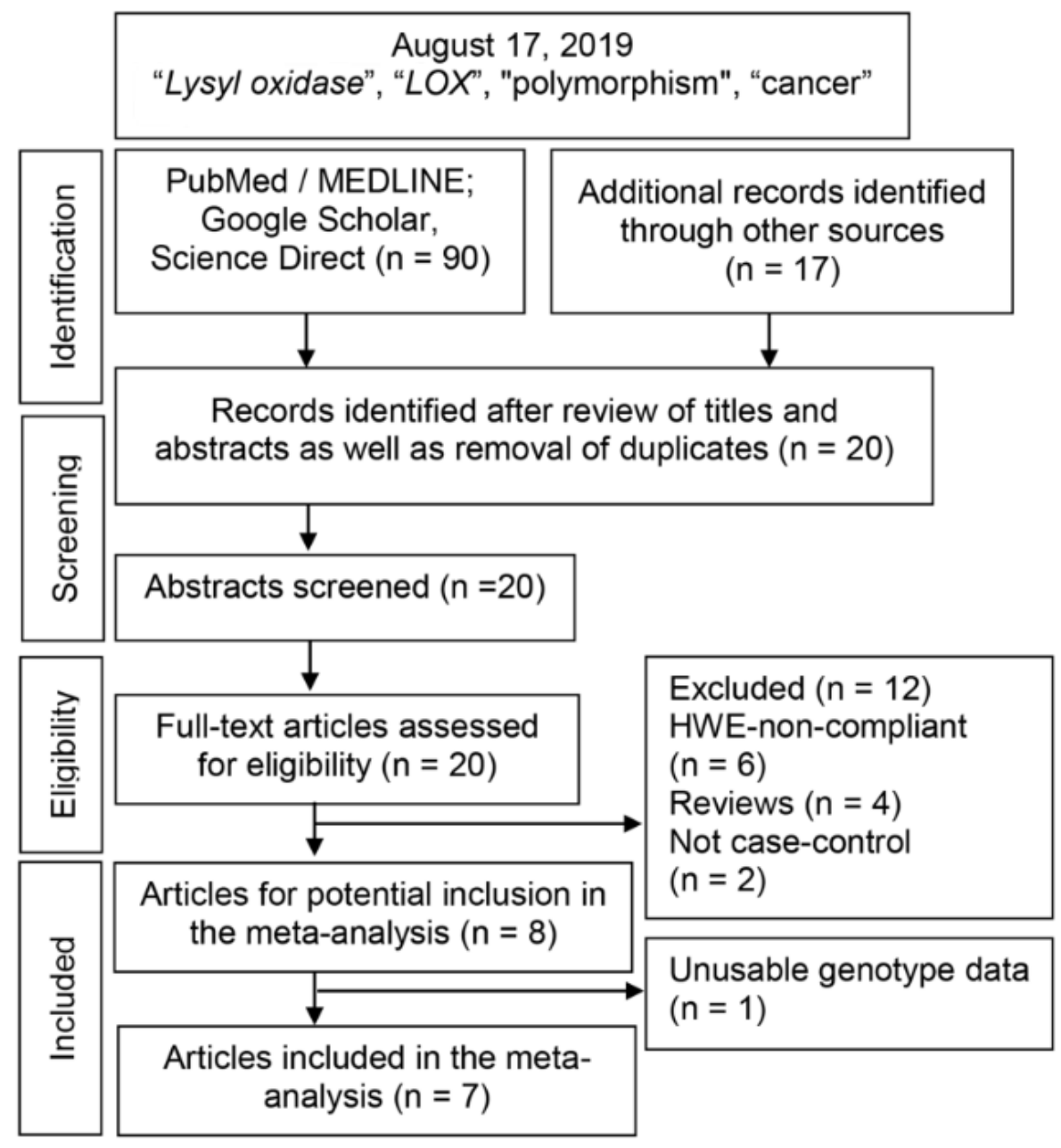

Figure 1

The selection process in a PRISMA (Preferred Reporting Items for Systematic Reviews and Meta-Analyses)-sanctioned flowchart. 


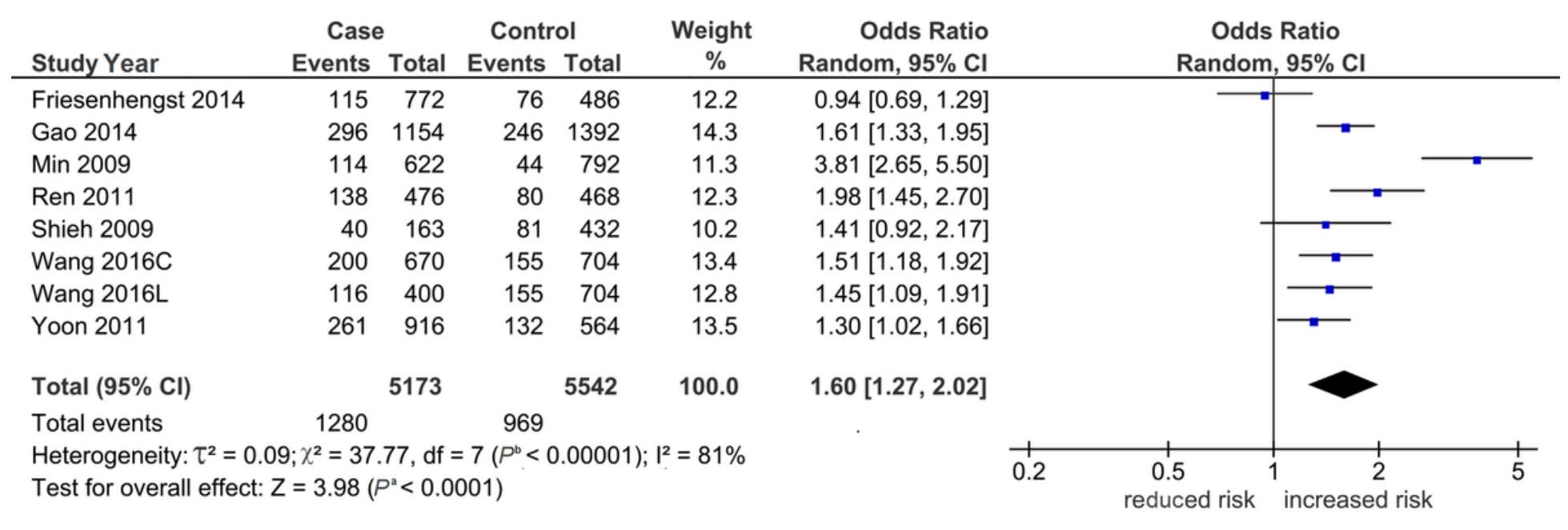

Figure 2

PRO forest plot, where the pooled outcome indicated significant increased risk (OR 1.60, 95\% Cl 1.27-2.02, Pa < 10-4) and heterogeneity (Pb < 10-5, I2 $=81 \%$ ).

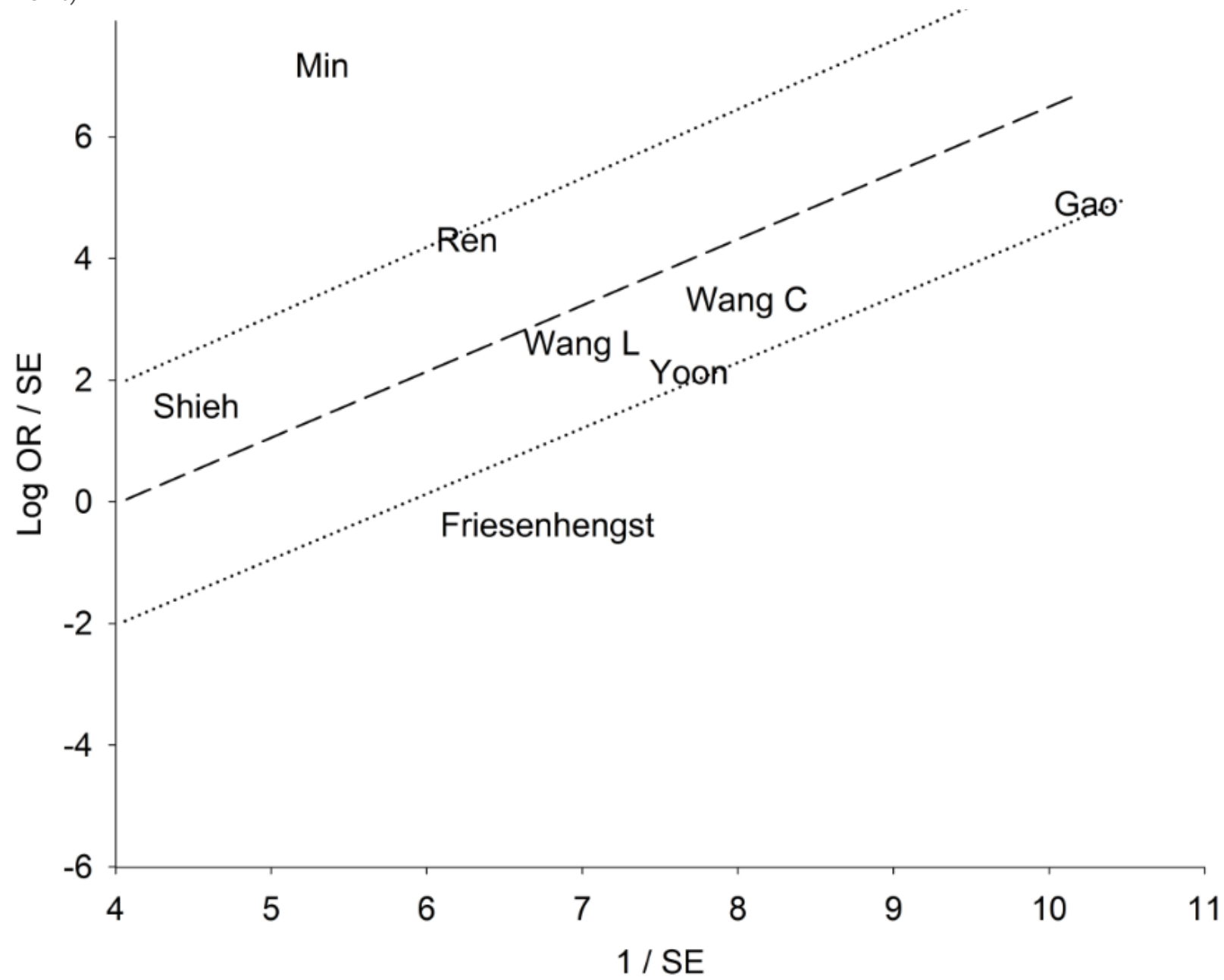

Figure 3

The Galbraith plot identified two studies $[27,29]$ as the outliers, located above and below the +2 and -2 confidence limits 


\begin{tabular}{|c|c|c|c|c|c|c|c|c|c|c|}
\hline \multirow{2}{*}{ Study Year } & Case & Total & $\begin{array}{l}\text { Contr } \\
\text { Events }\end{array}$ & $\begin{array}{l}\text { ol } \\
\text { Total }\end{array}$ & $\begin{array}{c}\text { Weight } \\
\%\end{array}$ & $\begin{array}{r}\text { Odds Ratio } \\
\text { Fixed, } 95 \% \mathrm{Cl}\end{array}$ & \multicolumn{4}{|c|}{$\begin{array}{c}\text { Odds Ratio } \\
\text { Fixed, } 95 \% \mathrm{Cl}\end{array}$} \\
\hline & 296 & 1154 & 246 & 1392 & 29.6 & $1.61[1.33,1.95]$ & & & -- & \\
\hline Ren 2011 & 138 & 476 & 80 & 468 & 10.2 & $1.98[1.45,2.70]$ & & & & \\
\hline Shieh 2009 & 40 & 163 & 81 & 432 & 6.0 & $1.41[0.92,2.17]$ & & & & \\
\hline Wang 2016C & 200 & 670 & 155 & 704 & 19.0 & $1.51[1.18,1.92]$ & & & $\longrightarrow$ & \\
\hline Wang 2016L & 116 & 400 & 155 & 704 & 14.3 & $1.45[1.09,1.91]$ & & & $\longrightarrow$ & \\
\hline Yoon 2011 & 261 & 916 & 132 & 564 & 20.9 & $1.30[1.02,1.66]$ & & & 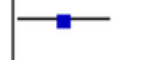 & \\
\hline Total $(95 \% \mathrm{Cl})$ & & 3779 & & 4264 & 100.0 & $1.53[1.38,1.70]$ & & & 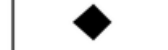 & \\
\hline Total events & 1051 & & 849 & & & & & & & \\
\hline $\begin{array}{l}\text { Heterogeneity: } \\
\text { Test for overall }\end{array}$ & $\begin{array}{l}7 \mathrm{df}=5( \\
=7.89(F\end{array}$ & $\begin{array}{l}\left(P^{\mathrm{b}}=0 .\right. \\
P^{\mathrm{a}}<0.0\end{array}$ & $\begin{array}{l}13) ;\left.\right|^{2}=0 \\
0001)\end{array}$ & & & & 0.2 & $\begin{array}{l}0.5 \\
\text { reduced risk }\end{array}$ & $\begin{array}{lr}1 & 2 \\
& \end{array}$ & 5 \\
\hline
\end{tabular}

Figure 4

The PSO outcome. 